\title{
COUNTABLE SUM THEOREM FOR LOCALLY CLOSED SETS
}

\section{Satya Deo and Dalip Singh Jamwal}

\begin{abstract}
In this paper we show that the countable sum theorem for locally closed sets, for sheaf theoretic cohomological dimension over a given ring $L$, holds in all perfectly normal spaces as well as in all locally compact spaces.
\end{abstract}

\section{Introduction}

Let dim denote the covering dimension of a normal space $X$. If $\left\{A_{n}\right\}_{n=1}^{\infty}$ is a family of closed subsets of $X$ such that $X=\bigcup_{n=1}^{\infty} A_{n}$, then it is well-known that

$$
\operatorname{dim} X=\sup _{n}\left\{\operatorname{dim} A_{n}\right\} .
$$

Such a result is usually known as the countable sum theorem for closed sets for the covering dimension dim [6]. If $\operatorname{dim}_{L}(X)$ denotes the sheaf theoretic cohomological dimension of the space $X$ over the ring $L$ ([2], p. 74), then the countable sum theorem for closed sets is also valid for $\operatorname{dim}_{L}$ [5]. Recall that a subset $Y$ of a space $X$ is said to be locally closed in $X$ if $Y$ is open in $\bar{Y}$ (the closure of $Y$ ). In particular, each open set and each closed set is locally closed. Whether or not the

Received 3 October 1983.

Copyright Clearance Centre, Inc. Serial-fee code: 0004-9727/84 $\$ \mathrm{~A} 2.00+0.00$. 
countable sum theorem for locally closed sets holds for sheaf theoretic cohomological dimension $\operatorname{dim}_{L}$, has been an open problem for quite some time. Such a result cannot be valid for the covering dimension dim, simply because the covering dimension $\operatorname{dim} U$ of an open subset $U$ of a normal space $X$ can be strictly greater than $\operatorname{dim} X$. For sheaf theoretic cohomological dimension $\operatorname{dim}_{L}$, however, $\operatorname{dim}_{L}(Y) \leq \operatorname{dim}_{L}(X)$ for any locally closed subset $Y$ of $X$, and hence to prove the countable sum theorem for locally closed sets for $\operatorname{dim}_{L}$, it suffices to show that if $X=\bigcup_{n=1}^{\infty} A_{n}$, where each $A_{n}$ is locally closed and $\operatorname{dim}_{L}\left(A_{n}\right) \leq k$ for each $n$, then $\operatorname{dim}_{L}(X) \leq k$. The objective of this paper is to prove the countable sum theorem for locally closed sets for sheaf theoretic cohomological dimension $\operatorname{dim}_{L}$ in a locally compact space. We also show that the same theorem is valid in a normal space $X$ in which every locally closed set is a generalized $F_{\sigma}$-set; in particular, it is valid in all perfectly normal spaces. In fact, we prove that in such a space countable sum theorem for closed sets is equivalent to the countable sum theorem for locally closed sets.

\section{Preliminaries}

First of all we recall Cartan's definition of cohomological $\phi$-dimension of a space $X$, where $\phi$ is a family of supports on $X$. For any ring $L$, let $A$ denote a sheaf of $L$-modules on $X$ and $H_{\phi}^{*}(X ; A)$ be the sheaf cohomology of $X$. with supports in $\phi$. Then the smallest integer $n$ (or $\infty$ ) such that $H_{\phi}^{i}(X ; A)=0$ for each $i>n$ and each sheaf $A$ of $L$-modules on $X$, is called the cohomological $\phi$-dimension of $X$ over the ring $L$ and is denoted by $\operatorname{dim}_{\phi, L}(X)$. It turns out that if $\phi, \psi$ are two paracompactifying families of supports on $X$ having the same extents, then $\operatorname{dim}_{\phi, L}(X)=\operatorname{dim}_{\psi, L}(X) \quad([2], p .74)$. Thus if $X$ admits a paracompactifying family $\phi$ of supports whose extent is $X$, then we can define the cohomological dimension of $X$ over the ring $L$, denoted by $\operatorname{dim}_{L}(X)$, to be $\operatorname{dim}_{\phi, L}(X)$. Locally paracompact spaces, which include all 
locally compact spaces and all paracompact spaces, form such a class for which $\operatorname{dim}_{L}(X)$ is always defined. However, if $\phi$ is not paracompactifying or its extent does not equal $X$, then $\operatorname{dim}_{\phi, L}(X)$ may turn out to be different from the desirable one ([3], [4]).

Now, let us recall that a subset $y$ of a topological space $X$ is said to be locally closed at a point $x \in Y$ if there is a neighbourhood $V$ of $x$ in $X$ such that $Y \cap V$ is a closed subset of the subspace $V$. $Y$ is said to be Zocally closed in $X$ if it is locally closed at each point of $Y$. A characterization of locally closed subsets is as follows ([1], p. 38).

PROPOSITION 2.1. For a subset $Y$ of a topological space $X$, the following properties are equivalent:

(a) $y$ is locally closed in $X$;

(b) $Y$ is the intersection of an open subset and a closed subset of $X$;

(c) $Y$ is open in $\bar{Y}$, the closure of $Y$ in $X$.

It is easy to see that

(i) the intersection of two locally closed sets is again locally closed, but the arbitrary intersection of locally closed sets need not be locally closed,

(ii) the union of two locally closed sets need not be locally closed, and

(iii) the complement of a locally closed set need not be locally closed.

We shall also need the following results.

THEOREM 2.2 (Subset Theorem ([2], p. 74)). If $x$ is Zocally paracompact (respectively, completely paracompact), then $\operatorname{dim}_{L}(X) \geq \operatorname{dim}_{L}(A)$ for any locally closed (respectively, arbitrary) subspace $A \subset X$.

THEOREM 2.3 (Countable Sum Theorem ([5], Corollary 4.6)). Let $X$ be a locally paracompact space and $\left\{F_{p} \mid p=1,2, \ldots\right\}$ be a countable closed covering of $X$. Then, for any ming $L$, 


$$
\operatorname{dim}_{L}(X)=\sup \left\{\operatorname{dim}_{L}\left(F_{p}\right) \mid p=1,2,3, \ldots\right\} .
$$

THEOREM 2.4 (Disjoint Sum Theorem ([5], Corollary 4.12)). Let $X$ be a locally paracompact space and $F$ be a closed subset of $X$. For any ring $L$, if $\operatorname{dim}_{L}(F) \leq n$ and $\operatorname{dim}_{L}(A) \leq n$ for each closed subset $A$ of $X$ disjoint from $F$, then $\operatorname{dim}_{L}(X) \leq n$.

\section{Main result}

First of all we show that in an arbitrary locally paracompact space $X$, the sum theorem for any two locally closed sets, and consequently for any finite number of locally closed sets holds. Then we show that in a normal space $X$ in which every locally closed set is a generalized $F_{\sigma}$-set, the countable sum theorem for closed sets is equivalent to the countable sum theorem for locally closed sets. In particular, it is valid in all perfectly normal spaces. Finally, we prove our main result, namely, the countable sum theorem for locally closed sets in a locally compact space. Whether or not such a theorem is valid for any paracompact space remains still open.

PROPOSITION 3.1. Let $X$ be any topological space and $A, B$ be two Zocally closed subsets of $X$ such that they cover $X$. Then, for any ring $L$,

$$
\operatorname{dim}_{L}(X)=\max \left\{\operatorname{dim}_{L}(A), \operatorname{dim}_{L}(B)\right\}
$$

Proof. Suppose $m=\max \left\{\operatorname{dim}_{L}(A), \operatorname{dim}{ }_{L}(B)\right\}$. Since $A$ is locally closed, $A$ is open in its closure $\bar{A}$ in $X$. Therefore, $\bar{A} \backslash A$ is closed in $\bar{A}$ and hence in $B$. But $\operatorname{dim}_{L}(B) \leq m$, so by the subset theorem $\operatorname{dim}_{L}(\bar{A} \backslash A) \leq m$. Now let $C$ be any closed subset of $\bar{A}$ disjoint from $\bar{A} \backslash A$. Again by the subset theorem, $\operatorname{dim}_{L}(C) \leq m$ and hence by the disjoint sum theorem, it follows that $\operatorname{dim}_{L}(\bar{A}) \leq m$. Similarly, we can show that $\operatorname{dim}_{L}(\bar{B}) \leq m$. Hence by the finite sum theorem for closed sets, we conclude that $\operatorname{dim}_{L}(X) \leq m$.

Using induction on $n$, we obtain the following: 
COROLLARY 3.2. Let $X$ be any topological space and $\left\{A_{1}, A_{2}, \ldots, A_{n}\right\}$ be a finite cover of $x$ by locally closed subsets of $X$. Then, for any ring $L$,

$$
\operatorname{dim}_{L}(X)=\max \left\{\operatorname{dim}_{L}\left(A_{i}\right) \mid i=1,2, \ldots, n\right\} .
$$

Now we shall show that if $X$ is the union of two subsets of $X$ one of which is locally closed and $\mathrm{dim}_{L}$ of both sets is finite, then $\operatorname{dim}_{L}(X)$ is also finite. In fact $\operatorname{dim}_{L}(X)$ coincides with the maximum of the tro.

PROPOSITION 3.3. Let $X$ be any topological space and let $X=A \cup B$, where $A$ is locally closed in $X$ and $B$ is any subset of $X$. Then, for any ring $L$,

$$
\operatorname{dim}_{L}(X)=\max \left\{\operatorname{dim}_{L}(A), \operatorname{dim}_{L}(B)\right\}
$$

Proof. Suppose $m=\max \left\{\operatorname{dim}_{L}(A), \operatorname{dim}_{L}(B)\right\}$. Since $A$ is locally closed in $X, A$ is open in its closure $\bar{A}$ in $X$. This means $\bar{A} \backslash A$ is closed in $\bar{A}$ and hence in $X$. Thus $\bar{A} \backslash A$ is also closed in $B$. By the subset theorem, $\operatorname{dim}_{L}(\bar{A} \backslash A) \leq \operatorname{dim}_{L}(B) \leq m$. For any closed subset $F$ of $\bar{A}$ disjoint from $\bar{A} \backslash A, \operatorname{dim}_{L}(E) \leq m$ and so by the disjoint sum theorem $\operatorname{dim}_{L}(\bar{A}) \leq m$. Now let $C$ be any closed subset of $X$ disjoint from $\bar{A}$. Then $C$ is clearly closed in $B$ and so by the subset theorem, $\operatorname{dim}_{L}(C) \leq m$. Hence by the disjoint sum theorem, $\operatorname{dim}_{L}(X) \leq m$.

COROLLARY 3.4. Let $X=\bigcup_{i=1}^{\infty} A_{i}$, where each $A_{i}$ is either open or closed. Then, for any ring $L$,

$$
\operatorname{dim}_{L}(X)=\sup \left\{\operatorname{dim}_{L}\left(A_{i}\right) \mid i \in N\right\}
$$

Proof. Suppose $\operatorname{dim}_{L}\left(A_{i}\right) \leq n$ for each $i$. Let $A$ be the union of all open sets and $B$ be the union of all closed sets of the family $\left\{A_{i}\right\}$. Then clearly $\operatorname{dim}_{L}(A) \leq n$ and by the countable sum theorem for closed sets, $\operatorname{dim}_{L}(B) \leq n$. Since $A$ is open, we can apply the above proposition to conclude that $\operatorname{dim}_{L}(X) \leq n$. 
COROLLARY 3.5. Let $X=\bigcup_{i=1}^{\infty} A_{i}$, where finitely many $A_{i}$ 's are locally closed and the rest of them are closed. Then, for any ring $L$,

$$
\operatorname{dim}_{L}(X)=\sup \left\{\operatorname{dim}_{L}\left(A_{i}\right) \mid i \in N\right\} \text {. }
$$

Proof. Again, suppose $\operatorname{dim}_{L}\left(A_{i}\right) \leq n$ for each $i$. Without loss of generality, we can assume that $A_{1}, A_{2}, \ldots, A_{k}$ are all locally closed and $A_{i}$ is closed for each $i>k$. By the countable sum theorem for closed sets, $\operatorname{dim}_{L}(B) \leq n$ where $B=\bigcup_{i>k} A_{i}$. Applying Proposition 3.3 to $A_{k}$ and $B$ we find that $\operatorname{dim}_{L}\left(A_{k} \cup B\right) \leq n$. Again applying the same arguments to $A_{k-1}$ and $A_{k} \cup B$, we obtain that $\operatorname{dim}_{L}\left(A_{k-1} \cup A_{k} \cup B\right) \leq n$.

Proceeding like this we obtain, after a finite number of steps, that

$$
\operatorname{dim}_{L}\left(A_{1} \cup \ldots \cup A_{k} \cup B\right)=\operatorname{dim}_{L}\left(\bigcup_{i=1}^{\infty} A_{i}\right) \leq n \text {. }
$$

Note that in a perfectly normal space each open set is $F_{\sigma}$ and hence each locally closed set is also $F_{\sigma}$. Also recall that a subset $M$ of a space $X$ is said to be a generalized $F_{\sigma}$-set if for each open set $U \supset M$ there exists a $F_{\sigma}$-set $F$ of $X$ such that $M \subset F \subset U$. In particular, a locally closed set $A$ in a perfectly normal space is a generalized $F_{\sigma}$ set.

Now we have

PROPOSITION 3.6. Let $X$ be a normal space and $\left\{A_{i} \mid i \in \mathbb{N}\right\}$ be a countable covering of $X$ by locally closed sets. Suppose each $A_{i}$ is a generalized $F_{\sigma}$-set. Then, for any ring $L$,

$$
\operatorname{dim}_{L}(X)=\sup \left\{\operatorname{dim}_{L}\left(A_{i}\right) \mid i \in \mathbf{N}\right\}
$$

Proof. In fact, we shall show that in such a case the countable sum theorem for locally closed sets is equivalent to the countable sum theorem for closed sets. By the subset theorem for $\operatorname{dim}_{L}, \operatorname{dim}_{L}\left(A_{i}\right) \leq \operatorname{dim}_{L}(X)$ 
for each $i$. Conversely, suppose $\operatorname{dim}_{L}\left(A_{i}\right) \leq n$ for each $i$. Now $A_{i}$ is locally closed in $X$ implies that $A_{i}=G_{i} \cap C_{i}$, where $G_{i}$ is open in $X$ and $C_{i}$ is closed in $X$ for each $i$. Since $A_{i} \subset G_{i}$ and each $A_{i}$ is a generalized $F_{\sigma}$-set, there exists a $F_{\sigma}$-set $F_{i}$ of $X$ such that $A_{i} \subset F_{i} \subset G_{i}$. Therefore

$$
\begin{aligned}
A_{i}=G_{i} \cap C_{i} & =E_{i} \cap C_{i} \\
& =\left(\begin{array}{cc}
\bigcup_{j=1}^{\infty} & F_{i, j}
\end{array}\right) \cap C_{i}=\bigcup_{j=1}^{\infty}\left(F_{i, j} \cap C_{i}\right)=\bigcup_{j=1}^{\infty} M_{i, j},
\end{aligned}
$$

where $M_{i, j}=F_{i, j} \cap C_{i}$ is closed in $X$ for each $i$ and $j$. Also $M_{i, j}$ is a closed subset of $A_{i}$ for each $j$ and so by the subset theorem, $\operatorname{dim}_{L}\left(M_{i, j}\right) \leq n$. Thus $\operatorname{dim}_{L}\left(M_{i, j}\right) \leq n$ for each $i$ and $j$. Therefore

$$
X=\bigcup_{i=1}^{\infty} A_{i}=\bigcup_{i=1}^{\infty}\left(\bigcup_{j=1}^{\infty} M_{i, j}\right)
$$

Hence by the countable sum theorem for closed sets, $\operatorname{dim}_{L}(X) \leq n$.

COROLLARY 3.7. In case $X$ is a perfectly normal space (for example, $X$ is metrizable) then the countable sum theorem for locally closed sets is valid for cohomological dimension $\operatorname{dim}_{L}$ over any ring $L$.

Now we have our main result.

THEOREM 3.8. Let $X$ be a locally compact (Hausdorff) space and $\left\{A_{n} \mid n \in N\right\}$ be a countable covering of $X$ by locally closed subsets of $X$. Then, for any ring i $L$,

$$
\operatorname{dim}_{L}(X)=\sup \left\{\operatorname{dim}_{L}\left(A_{n}\right) \mid n \in \mathrm{N}\right\} .
$$

Proof. By the local nature of $\operatorname{dim}_{L}$, and the subset theorem for locally closed subsets, we can assume, without loss of generality, that $X$ is compact. Since the subset theorem for $\operatorname{dim}_{L}$ holds for locally closed subsets, it suffices to show that if $\operatorname{dim}_{L}\left(A_{n}\right) \leq k$ for each $n \in \mathbb{N}$, then $\operatorname{dim}_{L}(X) \leq k$. Let us assume that $\operatorname{dim}_{L}(X)>k$. If, for each $n$, 
$\operatorname{dim}_{L}\left(\bar{A}_{n} \cup_{n}\right) \leq k$, then $\bar{A}_{n} \bigvee_{n}$ being closed in $\bar{A}_{n}$, it follows from the complementary sum theorem for $\operatorname{din}_{L}$ ([5], Corollary 4.10) that $\operatorname{dim}_{L}\left[A_{n}\right] \leq k$ for each $n \in N$. But, then by the countable sum theorem for closed sets, we find that $\operatorname{dim}_{L}(X) \leq k$, a contradiction. Let $n_{1}$ be the first natural number such that $\operatorname{dim}_{L}\left(A_{n_{1}} \backslash A_{n_{1}}\right)>k$. Now, for each $i<n_{1}, \operatorname{dim}_{L}\left(\bar{A}_{i}^{-}\right) \leq k$ and hence

$$
\left.\operatorname{dim}_{L}\left(\bigcup_{i<n_{1}}\left[A_{i} \cap \bar{A}_{n_{1}} \backslash A_{n_{1}}\right)\right]\right] \leq k
$$

Since $\left.\underset{i<n_{1}}{U}\left[A_{i} \cap \bar{A}_{n_{1}} \backslash A_{n_{1}}\right)\right]$ is closed in $\bar{A}_{n_{1}} \backslash A_{n_{1}}$, we can apply the disjoint sum theorem to obtain a closed set $B_{1}$ of $\bar{A}_{n_{1}}{ }_{A_{n_{1}}}$ which is disjoint from $A_{1}, A_{2}, \ldots, A_{n_{1}}$; that is, disjoint from $\underset{i \leq n_{1}}{\cup} A_{i}$ and $\operatorname{dim}_{L}\left(B_{1}\right\}>k$. Notice that $\left\{A_{p} n^{B_{1}}\right\}_{p>n_{1}}$ is a family of locally closed subsets of $B_{1}$ which covers $B_{1}$, and for each $p>n_{1}$, $\operatorname{dim}_{L}\left(A_{p} \cap B_{1}\right) \leq \operatorname{dim}_{L}\left(A_{p}\right) \leq k$. Replacing $X$ by $B_{1}$, the family $\left\{A_{n} \mid n \in N\right\}$ by $\left\{A_{p} \cap B_{1}\right\}_{p>n_{1}}$ and applying the same arguments as above we can find a $n_{2}>n_{1}$ and a closed subset $B_{2}$ of $B_{1}$ which is disjoint from $A_{1}, A_{2}, \ldots, A_{n_{1}}, \ldots, A_{n_{2}}$ and $\operatorname{dim}_{L}\left(B_{2}\right)>k$. Continuing this process by induction we find a decreasing sequence $\left\{B_{p}\right\}_{p \geq 1}$ of non-empty closed subsets of $X$ and a subsequence $\left\{n_{p}\right\}_{p \geq 1}$ of $N$ such that for each $p, B_{p}$ is disjoint from $A_{1}, A_{2}, \ldots, A_{n_{p}}, n_{p}>p$ and $\operatorname{dim}_{L}\left(B_{p}\right)>k$. Since each $B_{p}$ is a non-empty compact subset of $X, B=\bigcap_{p \geq 1} B_{p}$ must be non-empty and must be disjoint from $\underset{n \geq 1}{\mathrm{U}} A_{n}=X$. This is a contradiction. 


\section{References}

[1] Nicolas Bourbaki, Elements of mathematics. General topology (Hermann, Paris; Addison-Wesley, Reading, Massachussetts; Palo Alto; London; 1966).

[2] Glen E. Bredon, Sheaf theory (McGraw-Hill, New York, Toronto, London, 1968).

[3] Satya Deo, "One dimension manifold is of cohomological dimension two", Proc. Amer. Math. Soc. 52 (1975), 445-446.

[4] Satya Deo, "Cohomological dimension of a $n$-manifold is $n+1$ ", Pacific J. Math. 67 (1976), 155-160.

[5] Satya Deo and Ram Anugrah Shukla, "On cohomological dimension and the sum theorems", Acta Math. Acad. Sci. (to appear).

[6] Keiô Nagami, Dimension theory (Pure and Applied Mathematics, 37. Academic Press, New York and London, 1970).

\footnotetext{
Department of Mathematics,

University of Jammu,

Jammu 180001,

India.
} 